\title{
Is it justified to apply a modified Cabrol fistula in surgical repair of acute type A aortic dissection?
}

Hui Zhang, MD, Xijie Wu, MD, Guanhua Fang, MD, Zhihuang Qiu, MD, and Liang-wan Chen, MD

\section{ABSTRACT}

Objective: Open repair of acute type A aortic dissection frequently results in oozing from the suture lines. A modified fistula technique was developed to rapidly control oozing and allow closing the chest immediately and safely. The efficiency of this modified fistula technique in surgical repair of acute type A aortic dissection was evaluated.

Methods: This was a retrospective study. From January 2015 to December 2016, 172 patients with acute type A aortic dissection underwent emergency surgical repair in our center. Among them, 76 were treated with the modified Cabrol fistula technique (fistula group), and the others did not receive this modified technique (nonfistula group). The clinical data of all patients were retrospectively reviewed and analyzed.

Results: The preoperative data, cardiopulmonary bypass time, aortic crossclamp time, selective cerebral perfusion, and lower body arrest time of the 2 groups were similar. The chest closure time was shorter in the fistula group. The postoperative drainage, amount of red blood cell transfusion, incidence of reopening for hemostasis, mechanical ventilation support time, duration of intensive care unit stay, incidence of postoperative dialysis, and 30-day mortality were significantly lower in the fistula group than in the nonfistula group.

Conclusions: During surgical repair of acute type A aortic dissection, a modified Cabral fistula technique can rapidly control oozing and effectively improve the short-term outcomes. (J Thorac Cardiovasc Surg 2019;158:1307-14)

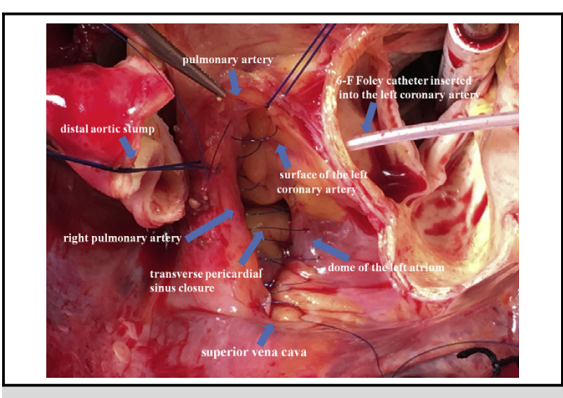

The transverse pericardial sinus was closed, and a Foley catheter was inserted for the guidewire.

\section{Central Message}

During surgical repair of acute type A aortic dissection, a modified Cabral fistula technique can be used to provide rapid control of oozing and effective improvement for the short-term outcomes.

\section{Perspective}

Surgical repair of acute type A aortic dissection with the modified Cabrol fistula technique, which includes suture closure of the transverse pericardial sinus, a pericardial patch coverage over the pericardial recess, and an opening made in the inferior inner side of the right atrium or in the left inner side of the superior vena cava, can control oozing and improve short-term outcomes.

See Commentary on page 1315.
Although meticulous surgical techniques and hemostatic agents have been widely used, oozing from suture lines frequently arises after an open repair of acute type A aortic dissection, mainly because the suture line is usually located

\footnotetext{
From the Department of Cardiac Surgery, Union Hospital, Fujian Medical University, Fuzhou, Fujian, China.

Funded by National Natural Science Foundation of China.

Received for publication Feb 27, 2018; revisions received Nov 25, 2018; accepted for publication Dec 25, 2018; available ahead of print Feb 6, 2019.

Address for reprints: Liang-wan Chen, MD, Department of Cardiac Surgery, Union Hospital, Fujian Medical University, Fuzhou, Fujian, 350001, China (E-mail: chenliangwan@tom.com).

0022-5223

Copyright (C) 2019 by The American Association for Thoracic Surgery. Published by Elsevier Inc. This is an open access article under the CC BY-NC-ND license (http:// creativecommons.org/licenses/by-nc-nd/4.0/).

https://doi.org/10.1016/j.jtcvs.2018.12.082
}

in the aortic segment involved by the dissection, the blood coagulability is decreased after the dissection process, and the cardiopulmonary bypass time is long. ${ }^{1-5}$ Oozing eventually can be controlled in the majority of cases, but pericardial blood aspiration and recovery of complete hemostasis take a long time. Furthermore, catastrophic oozing that cannot be controlled by conventional methods occasionally may occur, and it is usually

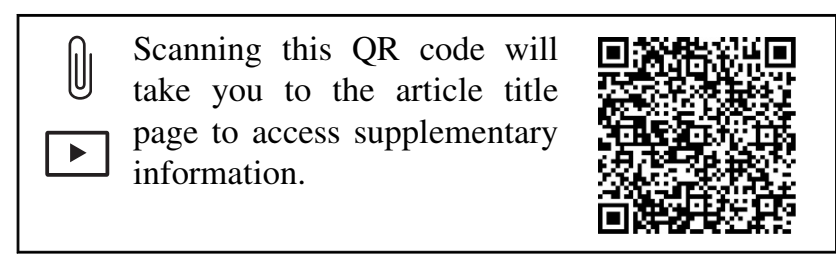




\section{Abbreviations and Acronyms \\ $\mathrm{CABG}=$ coronary artery bypass grafting \\ $\mathrm{CPB}=$ cardiopulmonary bypass \\ $\mathrm{EF}=$ ejection fraction \\ INR = international normalized ratio \\ $\mathrm{RBC}=$ red blood cell \\ $\mathrm{SCP}=$ selective cerebral perfusion}

unpredictable. $^{1,6,7}$ Therefore, an approach that can effectively control oozing and allow surgeons to close the chest immediately and safely is needed for the repair of acute type A aortic dissection.

The Cabrol shunt or Cabrol fistula, also known as a "perigraft-to-right atrial shunt," is a technique used for uncontrolled bleeding during graft replacement of ascending aortic and aortic root within the aneurysmal sac (inclusion technique). The fistula is constructed between the aneurysm wrap and the right atrial appendage for autotransfusion of the oozing into the circulation. ${ }^{8}$ It can turn an oozing situation into a clear surgical field that is completely dry of blood. Therefore, the Cabrol fistula has been proposed as an effective technique to control oozing in the surgical repair of acute type A aortic dissection. ${ }^{6,7}$ Compared with the inclusion technique, the open technique with resection of all aortic walls allows more precise suturing, easier access to bleeding sites, and more rapid hemostasis. Therefore, the majority of the surgeons have adopted the open technique instead of the inclusion technique in surgical repair of acute type A aortic dissection. ' However, the classic Cabrol fistula cannot be used in an open technique because there is no option to wrap the graft with the aortic wall. ${ }^{6}$ During surgical repair of acute type A aortic dissection with the open technique, a modified Cabrol fistula technique to control oozing was performed in our center. ${ }^{10}$ The procedure includes suture closure of the transverse pericardial sinus, a pericardial patch coverage over the pericardial recess between the superior vena cava and the pulmonary artery, and an opening in the roof of the right atrium or in the left inner side of the superior vena cava. The present report retrospectively evaluates the efficacy of this modified fistula technique.

\section{MATERIALS AND METHODS}

The present retrospective study was approved by the Ethics Committee of the Union Hospital, Fujian Medical University.

\section{Patients}

From January 2015 to December 2016, 172 patients with acute type A aortic dissection underwent emergency surgical repair in our center. The preoperative diagnosis was made on contrast-enhanced computed tomography angiography and echocardiography. All operations were performed within 4 hours after diagnosis. A total of 76 patients underwent closure of the transverse pericardial sinus and were treated with a modified Cabrol fistula procedure (fistula group, $\mathrm{n}=76$ ), whereas others were not treated with the modified fistula procedure (nonfistula group, $n=96$ ). All operations were performed by the same surgeon (L-w.C.). The decision to perform this technique was at the second surgeon's discretion and was dependent on patients' conditions: (1) patients with long-term treatment of aspirin or a preoperative thrombolysis for a presumed myocardial infarction; (2) menstrual women; and (3) patients with a dissected ascending aortic wall with severe edema. The preoperative characteristics of the patients are shown in Table 1.

\section{Surgical Procedure}

A median sternotomy was performed, and the total cardiopulmonary bypass was established by 2 venous cannulas through the right atrium and arterial return cannulas inside both femoral and right axillary arteries. When the nasopharyngeal temperature decreased to $32^{\circ} \mathrm{C}$, the ascending aorta was clamped at the position proximal to the innominate artery and transected just above the sinotubular junction. Myocardial protection was accomplished by multiple infusion of cold blood cardioplegia $\left(4^{\circ} \mathrm{C}\right)$ into the coronary ostia. After careful inspection of the aortic root, valve, and coronary ostia, the aortic root manipulations were performed, including repair of the sinus Valsalva, Bentall procedure, and coronary artery bypass grafting. Subsequently, the repaired aortic root was anastomosed to a straight Dacron tube graft. All distal aortic anastomoses were performed by the open technique with the use of moderate hypothermic circulatory arrest $\left(20^{\circ} \mathrm{C}-25^{\circ} \mathrm{C}\right)$ in combination with cold selective antegrade cerebral perfusion at a rate of 8 to $10 \mathrm{~mL} \cdot \mathrm{kg}^{-1} \cdot \mathrm{min}^{-1}$.

In case of arch repair, a total arch replacement with a 4-branched Dacron tube graft combined with a stented elephant trunk implantation or an open triple-branched stent graft implantation was performed as previously described. ${ }^{11,12}$ The indications of the total arch repair are acute Stanford type A aortic dissection with (1) the intimal tear located in transverse arch or proximal descending aorta that could not be resected by hemiarch replacement; (2) marked involvement of the arch vessels; (3) Marfan syndrome, and (4) age less than 55 years. Patients for total arch repair were treated with open triple-branched stent graft placement technique if the diameters of the native aortic arch and arch vessels and the distances between 2 neighboring arch vessels (measured by preoperative 3-dimensional computed tomography) matched the corresponding size of the triple-branched stent grafts available: The diameters of the native aortic arch and arch vessels were $10 \%$ to $20 \%$ smaller than the diameters of the corresponding stent grafts, and the distances between the 2 neighboring arch vessels were equal to the distances between the 2 corresponding sidearm stent grafts. If the patients had an indication for total arch repair but were not eligible for open triple-branched stent graft placement, total arch replacement with a 4-branched Dacron tube graft combined with a stented elephant trunk implantation was applied.

The patients in the fistula group underwent an additional modified Cabrol shunt procedure. The details of the procedure have been reported. ${ }^{10}$ Briefly, patients had their transverse pericardial sinus closed before ascending graft implantation. This transverse pericardial sinus closure was completed by direct suture approximation or using a small autologous or bovine pericardial patch. During the closure, a 6-F Foley catheter was inserted into the left coronary artery and served as a guidewire to prevent the coronary artery from injury (Figure 1, $A$ ). Before the termination of cardiopulmonary bypass, a bovine pericardial patch was appropriately tailored to cover the pericardial recess between the superior vena cava and the pulmonary artery after no blood spurting was seen (Figure $1, B$ ). According to the aortic repaired extent (ascending, hemiarch, or total arch replacement), a continuous 4-0 Prolene suture was used to sew the bovine pericardial patch to the following surrounding tissues: innominate vein, arch, pulmonary artery, right ventricular free wall overlying the aortic root, roof of the right atrium, left inner side of the superior vena cava, and pericardial fold overlying the distal ascending aorta (Figure 2, A-C). The suture was 
TABLE 1. Preoperative characteristics

\begin{tabular}{|c|c|c|c|c|}
\hline Characteristics & Fistula group $n=76$ & Nonfistula group $n=96$ & $t$ or $z$ test, or chi-square test & $P$ value \\
\hline Male & $51(67.1 \%)$ & $67(69.8 \%)$ & 0.01 & .94 \\
\hline Age, y & $51.3 \pm 11.0$ & $53.7 \pm 10.7$ & -1.38 & .17 \\
\hline Marfan syndrome & $17(22.4 \%)$ & $21(21.9 \%)$ & 0.01 & .94 \\
\hline Hypertension & $57(75.0 \%)$ & $70(72.9 \%)$ & 0.10 & .76 \\
\hline Diabetes & $5(6.6 \%)$ & $8(8.3 \%)$ & 0.19 & .67 \\
\hline Chronic renal dysfunction & $1(1.3 \%)$ & $2(2.1 \%)$ & & $1.00^{*}$ \\
\hline Previous open heart surgery & $1(1.3 \%)$ & $0(0.0 \%)$ & & $.44^{*}$ \\
\hline History of cerebrovascular event & $2(2.6 \%)$ & $3(3.1 \%)$ & & $1.00^{*}$ \\
\hline Acute heart failure & $2(2.6 \%)$ & $1(1.0 \%)$ & & $.59 *$ \\
\hline Acute renal dysfunction & $2(2.6 \%)$ & $0(0.0 \%)$ & & $.19^{*}$ \\
\hline Coronary heart disease & $0(0.0 \%)$ & $3(3.1 \%)$ & & $.26^{*}$ \\
\hline Hypoxemia & $4(5.3 \%)$ & $3(3.1 \%)$ & & $.70^{*}$ \\
\hline Prothrombin time, $\mathrm{s}$ & $14.0(13.2,15.0)$ & $13.8(13.2,14.8)$ & -1.22 & .22 \\
\hline INR & $1.1(1.0,1.2)$ & $1.1(1.0,1.2)$ & -0.56 & .58 \\
\hline $\mathrm{EF}, \%$ & $64.4(60.1,67.0)$ & $63.1(59.8,67.4)$ & -0.13 & .26 \\
\hline \multicolumn{5}{|l|}{ Pericardial effusion } \\
\hline Moderate to severe & $8(10.5 \%)$ & $8(8.3 \%)$ & 0.24 & .62 \\
\hline \multicolumn{5}{|l|}{ Aortic regurgitation } \\
\hline Moderate to severe & $25(32.9 \%)$ & $30(31.3 \%)$ & 0.05 & .82 \\
\hline The time from beginning of study to surgery, $m$ & $15.0(8.3,20.8)$ & $16.0(10.0,21.0)$ & -0.52 & .61 \\
\hline
\end{tabular}

$I N R$, International normalized ratio; $E F$, ejection fraction. *Fisher exact test.

initiated at the top of the bovine patch, with 1 arm clockwise from the pulmonary artery down to the right ventricular free wall. The other arm was placed at an opposite direction from the pericardial fold down to the superior vena cava. Two arms were finally tied at the junction between the superior vena cava and the right atrium. Before completion of the patch attachment, an 8- to $10-\mathrm{mm}$ opening was made in the roof of the right atrium or the left inner side of the superior vena cava and a Foley catheter was immediately inserted into the right atrium through this opening for temporary obstruction (Figure 1,C). This catheter was withdrawn just before the suture was tied (Video 1 ).

\section{Outcome Measures}

The medical records of the patients were retrospectively retrieved. Patient survival was confirmed by regular interviews in the hospital outpatient department or by telephone interviews conducted according to a standardized questionnaire. The date of the last follow-up was December 1, 2017.

The primary outcomes included the postoperative drainage, amount of red blood cell transfusion, and incidence of reopening for bleeding or oozing. The secondary outcomes included ventilation support period, intensive care unit stay duration, and 30-day mortality. Propensity score matching analysis for the preoperative characteristics was performed.

\section{Statistical Analysis}

SPSS 24.0 (SPSS Inc, Chicago, Ill) was used for statistical analysis. Normally distributed data were expressed as mean \pm standard deviation and compared using the $t$ test. Data that were not normally distributed were presented as median (2.5th-97.5th percentile) and compared using the Wilcoxon rank-sum test. The categoric variables were presented as percentage and analyzed with chi-square or Fisher exact test to indicate statistical significance.

\section{RESULTS}

\section{Baseline Characteristics}

The operative data of the 2 groups are shown in Table 2. There were no significant differences in terms of aortic root procedures, arch repair techniques, and concomitant surgeries between the 2 groups. The cardiopulmonary bypass time, aortic crossclamp time, selective cerebral perfusion and lower body arrest time, and lowest rectal temperature were also similar between the 2 groups. The chest closure time in the fistula group was shorter than in the nonfistula group $(P<.05)$.

\section{Outcomes}

A total of 6 patients in the fistula group and 8 patients in the nonfistula group were lost during the follow-up. The average durations of the follow-up time were $28 \pm$ 8.4 months for the fistula group and $27 \pm 11.8$ months for the nonfistula group.

\section{Primary Outcomes}

The postoperative data are shown in Table 3. The average postoperative drainage volume was $357.7 \pm 161.1 \mathrm{~mL}$ in the fistula group and $722.0 \pm 305.6 \mathrm{~mL}$ in the nonfistula group $(P<.0001)$. The average volume of packed red cells transfused in the fistula group and the nonfistula group was 

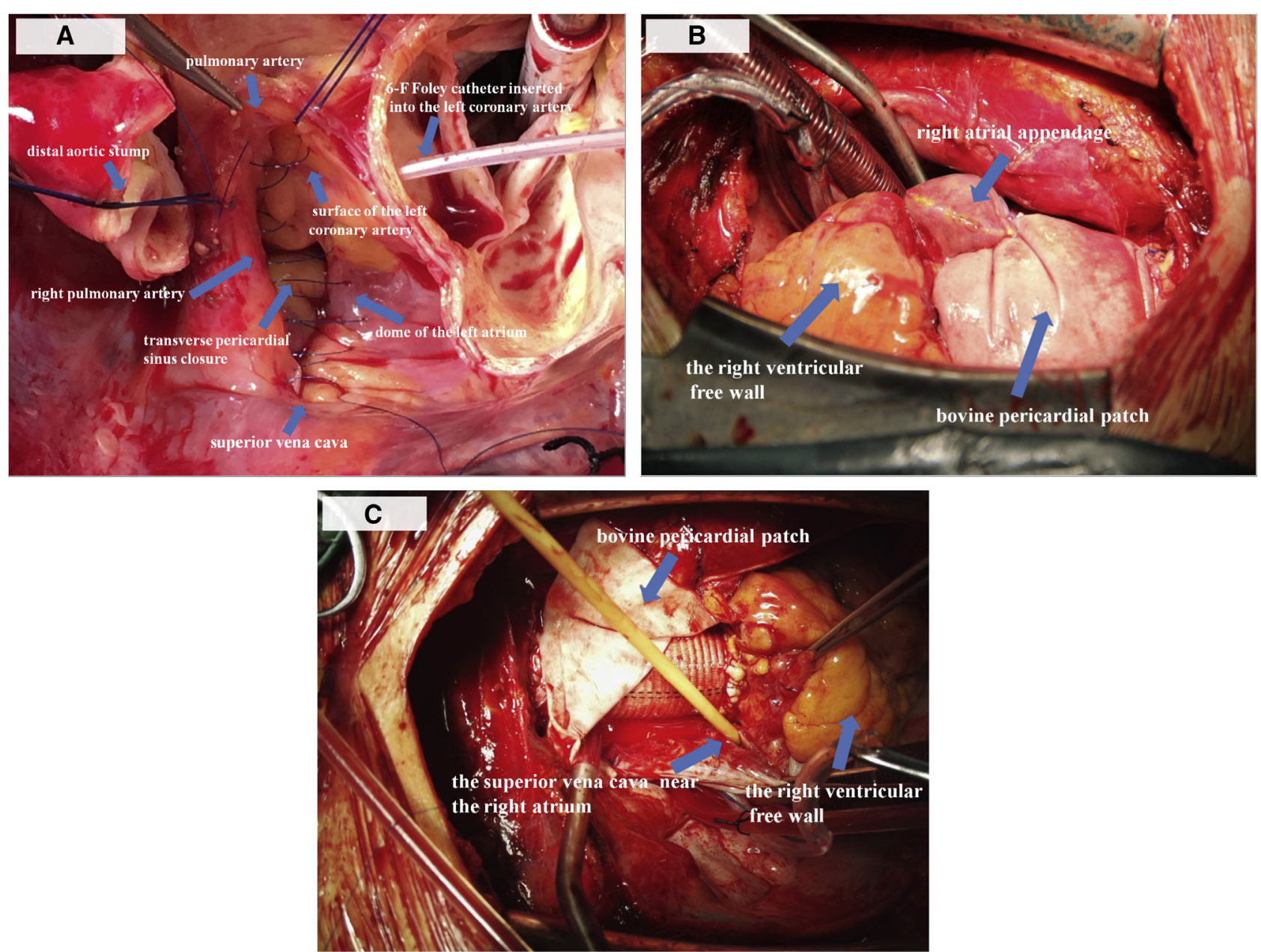

FIGURE 1. A-C, Representative photographs of a 70-year-old patient with acute type A aortic dissection undergoing ascending replacement. A, The transverse pericardial sinus closure was completed by direct suture between the left atrial roof and the pericardium overlying the right pulmonary artery. During the closure, a 6-F Foley catheter was inserted into the left coronary artery and served as a guidewire for keeping the coronary artery from damage. B, Before the cardiopulmonary bypass was stopped, a bovine pericardial patch was appropriately placed to cover the pericardial recess between the superior vena cava and the pulmonary artery after no spurt bleeding was found. C, After the completion of the patch attachment, an 8- to 10-mm opening was made in the inferior inner side of the right atrium and a Foley catheter was immediately inserted into the right atrium through this opening for temporary obstruction. This catheter was withdrawn just before the suture was tied.

$497.4 \pm 127.5 \mathrm{~mL}$ and $770.8 \pm 93.0 \mathrm{~mL}$, respectively $(P<.05)$. No patient needed reopening for oozing or bleeding in the fistula group, whereas 9 patients were reopened in the nonfistula group $(0.0 \%$ vs $9.4 \%, P=.005)$.

\section{Secondary Outcomes}

The postoperative mechanical ventilation support period and duration of intensive care unit stay were shorter in the fistula group compared with the nonfistula group (50.1 \pm 29.2 hours vs $72.3 \pm 70.4$ hours, $P=.0065$; $4.3 \pm 2.7$ days vs $8.1 \pm 7.5$ days, $P<.0001$, respectively). The incidence of postoperative dialysis was lower in the fistula group compared with the nonfistula group $(1.3 \%$ vs $15.6 \%, P=.001)$. No permanent neurologic dysfunction, paraplegia, or paralysis occurred in both groups. Although the incidence of temporary neurologic dysfunction and pulmonary infection tended to be higher in the nonfistula group, the difference did not reach statistic significance. The length of hospital stay and the 30-day mortality were lower in the fistula group compared with those in the nonfistula group.

In the fistula group, echocardiography and contrastenhanced computed tomographic angiography indicated that all fistulas were closed spontaneously with thrombus accumulation inside the perigraft compartments in a few days postoperation. The complications from this thrombus accumulation, namely, compression of the graft, right atrium, and superior vena cava, were not observed. No 

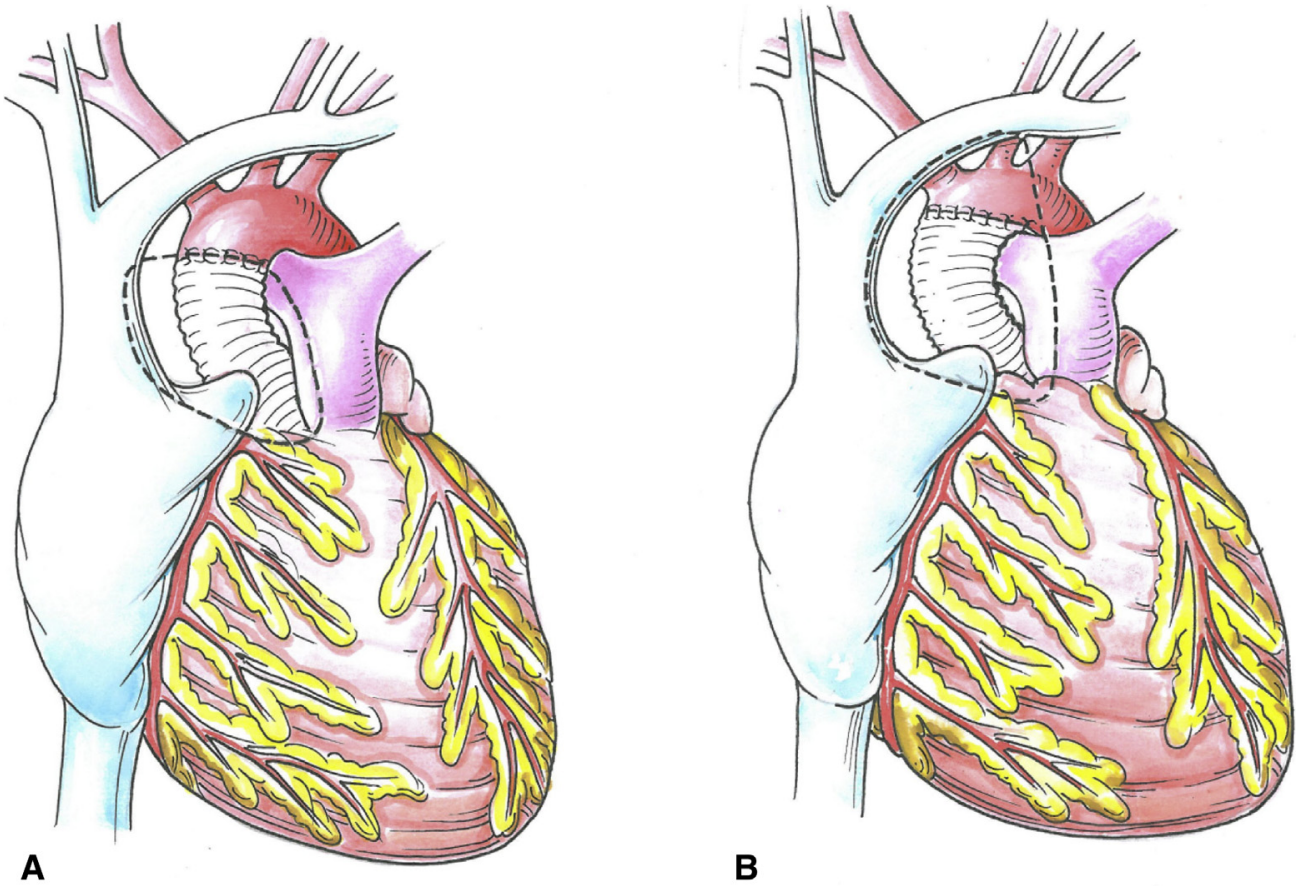

B

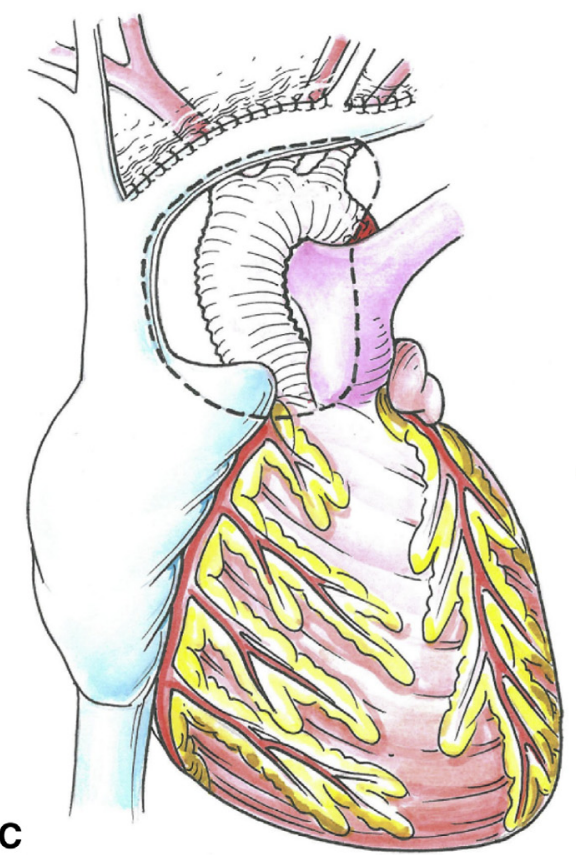

FIGURE 2. A-C, The extent of the bovine pericardial path coverage according to the aortic repaired extent (indicated by imaginary line). A, The top of the patch was sutured to the pericardial fold overlying the distal ascending aorta when only ascending replacement or ascending replacement combined with open placement of triple-branched stent graft was performed. B, The patch top was attached to the innominate vein when the hemiarch replacement was performed. C, During total arch replacement with a 4-branched Dacron tube graft combined with a stented elephant trunk implantation, the patch top was attached to the inferior side of the innominate vein and the upper side of the innominate vein was sutured to the upper mediastinal tissue.

patient developed heart failure induced by vast shunt flow. Similar results were found with propensity score matching analysis (Tables E1 and E2).

Two patients died in the fistula group (of cerebral hemorrhage and acute cardiac infarction, respectively), and 3 patients died in the nonfistula group ( 2 of cerebral hemorrhage and 1 of infection, respectively) during the follow-up period. All late deaths were not related to aortic events and the use of the modified fistula technique. 


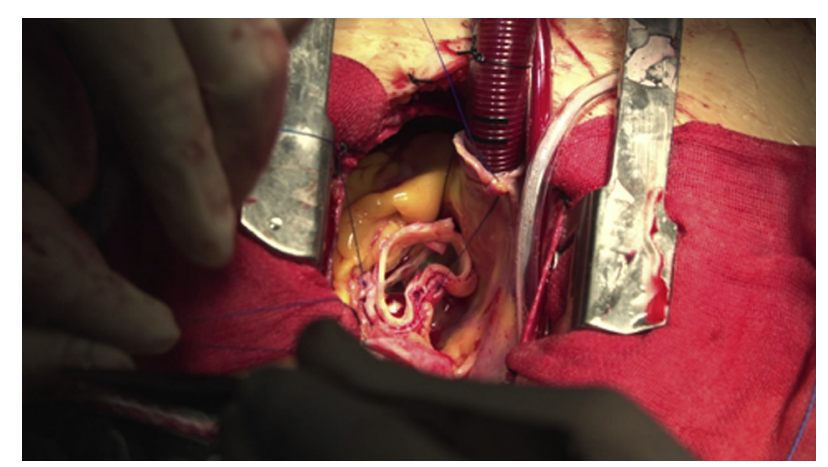

VIDEO 1. Before the ascending aortic graft implantation, the transverse pericardial sinus closure was completed by using a small bovine pericardial patch. A 5-0 Prolene suture was used to sew the patch to the surrounding structures: the fatty epicardium on the left main coronary artery, posterior adventitial material of the main pulmonary artery, pericardium overlying the right pulmonary artery and the left atrial roof. To prevent the left coronary artery from damage during the closure, a 6-F Foley catheter was inserted into the left coronary artery and served as a guidewire. Before the termination of the cardiopulmonary bypass, an appropriately trimmed sheet of bovine pericardium was sutured to surrounding structures to cover the pericardial recess between the superior vena cava and the pulmonary artery by 4-0 Prolene suture if no spurt bleeding was present. Before completion of the patch attachment, an 8- to 10-mm opening was made in the inferior inner side of the right atrium. A 10-F Foley catheter was inserted into the right atrium through this opening for temporary obstruction and was withdrawn just before the suture was tied. Video available at: https://www.jtcvs.org/article/S0022-5223(19)30009-1/fulltext.

\section{DISCUSSION}

The primary purpose of the use of our modified Cabrol fistula was to control the intractable bleeding during the surgical repair of acute type A aortic dissection with the open technique. Because it is difficult to close the transverse pericardial sinus with sutures after the grafts implantation, this transverse pericardial sinus closure is prophylactic performance. Furthermore, we cannot predict which patients will bleed after surgical repair. Therefore, this prophylactic transverse pericardial sinus closure and our modified Cabrol fistula was usually performed in patients with risk factors for severe bleeding after acute type A aortic dissection. The clinical results of our application of the modified Cabrol fistula showed that it provides primary and definite sternal closure, avoids the detrimental effects of a second pump run and continued bleeding, and consequently improves the early outcome. Enlightened by this satisfactory early result and realizing that our prophylactic transverse pericardial sinus closure is harmless and requires only 1 to 2 minutes, some surgeons routinely apply the modified Cabrol fistula in surgical repair of acute type A aortic dissection for the purpose of better early outcome. Our present study aims to demonstrate whether routine application of the modified Cabrol in surgical repair of acute type A aortic dissection is worthwhile.

The Cabrol fistula can collect the oozing blood and create a connection between the compartment and the venous system that enables autotransfusion of the oozing into the circulation. ${ }^{8}$ The present modified technique has 2 advantages compared with the classic Cabrol fistula technique and its modifications: (1) the closure of the transverse sinus before graft implantation, which is required for the construction of a modified new leak-proof perigraft compartment; and (2) the construction of an opening in the inferior inner side of the right atrium or in the left inner side of the superior vena cava, which can effectively transfuse the oozing into the circulation.

The use of an open technique for surgical repair of acute type A aortic dissection makes the construction of the

TABLE 2. Operative data

\begin{tabular}{|c|c|c|c|c|}
\hline Characteristics & Fistula group $n=76$ & Nonfistula group $n=96$ & $\begin{array}{c}t \text { or } z \text { test, or } \\
\text { chi-square }\end{array}$ & $P$ value \\
\hline \multicolumn{5}{|l|}{ Aortic root procedure } \\
\hline 1. Sinus repair & $53(69.7 \%)$ & $67(69.8 \%)$ & 0.00 & .99 \\
\hline 2. Bentall procedure & $18(23.7 \%)$ & $23(24.0 \%)$ & 0.00 & .97 \\
\hline \multicolumn{5}{|l|}{ Arch repair } \\
\hline 1. Total replacement with stented elephant trunk & $17(22.4 \%)$ & $21(21.9 \%)$ & 0.01 & .94 \\
\hline 2. Triple-branched stent graft implantation & $26(34.2 \%)$ & $39(40.6 \%)$ & 0.74 & .39 \\
\hline \multicolumn{5}{|l|}{ Concomitant procedure } \\
\hline 1. Mitral repair & $2(2.6 \%)$ & $1(1.0 \%)$ & & $.58 *$ \\
\hline 2. $\mathrm{CABG}$ & $1(1.3 \%)$ & $3(3.1 \%)$ & & $.63^{*}$ \\
\hline CPB time, $\min$ & $135.0(120.0,146.8)$ & $133.0(121.3,154.3)$ & -0.04 & .97 \\
\hline Crossclamp time, $\min$ & $47.0(40.3,53.5)$ & $48.0(42.0,54.0)$ & -0.52 & .60 \\
\hline SCP time, $\min$ & $10.0(9.0,12.0)$ & $10.0(9.0,12.0)$ & -0.79 & .43 \\
\hline Lowest rectal temperature, ${ }^{\circ} \mathrm{C}$ & $23.5 \pm 1.3$ & $23.7 \pm 1.2$ & 0.97 & .34 \\
\hline Chest closure time, min & $59.3 \pm 13.8$ & $85.1 \pm 26.4$ & 8.26 & $<.0001$ \\
\hline
\end{tabular}

$C A B G$, Coronary artery bypass grafting; $C P B$, cardiopulmonary bypass; $S C P$, selective cerebral perfusion. *Fisher exact test. 
TABLE 3. Postoperative data

\begin{tabular}{|c|c|c|c|c|}
\hline Characteristics & Fistula group $n=76$ & Nonfistula group $n=96$ & $t$ or $z$ test, or chi-square & $P$ value \\
\hline Postoperative drainage, $\mathrm{mL}$ & $342.5(232.5,450.0)$ & $685.0(476.0,890.0)$ & -8.24 & $<.0001$ \\
\hline $\mathrm{RBC}$ transfusion, $\mathrm{mL}$ & $500.0(400.0,600.0)$ & $800.0(600.0,900.0)$ & -8.64 & $<.0001$ \\
\hline Reopening for bleeding or oozing & $0(0.0 \%)$ & $9(9.4 \%)$ & & $.005^{*}$ \\
\hline Ventilation support, $\mathrm{h}$ & $45.0(28.0,60.0)$ & $48.0(20.0,91.8)$ & -0.78 & .44 \\
\hline Intensive care unit stay, $d$ & $4.0(2.0,6.0)$ & $5.5(4.0,9.0)$ & -4.06 & $<.0001$ \\
\hline Dialysis & $1(1.3 \%)$ & $15(15.6 \%)$ & 10.29 & .001 \\
\hline Low cardiac output syndrome & $1(1.3 \%)$ & $2(2.1 \%)$ & & $1.00 *$ \\
\hline Sepsis & $2(2.6 \%)$ & $4(4.2 \%)$ & & $.69 *$ \\
\hline Temporary neurologic dysfunction & $4(5.3 \%)$ & $10(10.4 \%)$ & 1.50 & .22 \\
\hline Length of stay, $d$ & $20.0(16.0,25.8)$ & $20.0(15.0,28.8)$ & -0.02 & .98 \\
\hline 30-d mortality & $2(2.6 \%)$ & $11(11.5 \%)$ & 4.73 & .03 \\
\hline
\end{tabular}

$R B C$, Red blood cell. *Fisher exact test.

classic perigraft compartment with native aortic wall impossible. Therefore, several modified perigraft compartments have been developed. ${ }^{1,6,13-16}$ These modifications are completed by the patch wrap technique or the pericardial recess coverage technique. In the patch wrap technique, a perigraft compartment is created by the placement of an autologous pericardium or a bovine pericardial patch that is wrapped around the graft. ${ }^{13-15}$ Because the patch is usually sutured to the proximal and distal anastomoses, it is impossible to wrap all suture lines inside the perigraft compartment. Furthermore, it is difficult to suture a pericardial patch posterior to the graft after the completion of the anastomoses between the graft and the aortic stumps. In the pericardial recess coverage technique, a perigraft compartment is formed with pericardial patch coverage over the pericardial recess between the superior vena cava and the pulmonary artery. ${ }^{1,16}$ This perigraft compartment can wrap all the graft and suture lines, but there is leaking of the blood from the transverse pericardial sinus. It is impossible to close the transverse pericardial sinus with sutures after the implantation of the graft. Consequently, the pericardial recess technique has only been used in reoperation where the transverse pericardial sinus is usually obliterated by postoperative adhesion. ${ }^{16}$ In patients receiving primary sternotomy, placement of an oversized piece of autologous pericardium and the injection of fibrin glue inside the transverse pericardial sinus were performed by some surgeons to close the transverse pericardial sinus. ${ }^{16}$ However, this packing with the pericardium cannot effectively prevent bleeding flowing into the posterior pericardium, whereas it usually compresses the left atrium. In the present modification, the transverse pericardial sinus is closed with sutures before the graft implantation. Thus, the compartment created by the pericardial recess coverage technique is leak-proof and safer.
In the classic Cabrol fistula and its modifications, the connection between the perigraft compartment and the venous system is established by direct anastomosis or a small graft, which is interposed. ${ }^{1,6,13}$ However, in the present modified Cabrol fistula technique, the connection is established by an opening in the roof of the right atrium or in the left inner side of the superior vena cava. Neither anastomosis nor a small interposed graft is necessary for this connection. Therefore, the current connection is simpler.

During the open repair of acute type A aortic dissection, oozing from the suture line occurs frequently and is usually uncontrollable before hemostasis is established. With our modified Cabrol fistula technique, all suture lines are wrapped inside the compartment and the blood from the suture line is continuously directed to the circulation. Therefore, immediate control of the oozing and dry surgical field is achieved. Consequently, a shorter time is needed for chest closure.

In addition, the postoperative drainage, amount of red blood cell transfusion, incidence of reopening for bleeding or oozing, postoperative mechanical ventilation support period, duration of intensive care unit stay, incidence of postoperative dialysis, length of hospital stay, and 30-day mortality were decreased in the patients treated by the modified Cabrol fistula technique. Thus, the current modified technique could improve the short outcome of the open repair of the acute type A aortic dissection due to the marked reduction of the postoperative blood loss and the prevention of red cell damage and destruction and plasma protein due to prolonged pericardial aspiration. Therefore, our modified Cabrol fistula technique is an appealing alternative technique for the control of oozing.

The long-term outcomes and potential disadvantages of our modified Cabrol fistulas still need to be determined. 
In the present study, all fistulas closed spontaneously with thrombus accumulation inside the perigraft compartments. No complications from thrombus accumulation were observed, and echocardiographic examination did not demonstrate compression of the graft, right atrium, or superior vena cava in the follow-up. No heart failure due to vast shunt flow occurred.

\section{CONCLUSIONS}

In the surgical repair of acute type A aortic dissection with an open technique, the present modified Cabrol fistula technique can effectively control oozing and improve short-term outcomes. Although the preliminary outcomes are encouraging, further multicenter randomized controlled trials are necessary to fully evaluate the efficacy of this technique.

\section{Conflict of Interest Statement}

Authors have nothing to disclose with regard to commercial support.

\section{References}

1. Toole JM, Stroud MR, Ikonomidis JS. Salvage periaortic pericardial baffle equalizes mortality in bleeding patients undergoing aortic surgery. J Thorac Cardiovasc Surg. 2014;148:151-5.

2. Leontyev S, Legare JF, Borger MA, Buth KJ, Funkat AK, Gerhard J, et al. Creation of a scorecard to predict in-hospital death in patients undergoing operations for acute type A aortic dissection. Ann Thorac Surg. 2016;101:1700-6.

3. Kondoh H, Satoh H, Daimon T, Tauchi Y, Yamamoto J, Abe K, et al. Outcomes of limited proximal aortic replacement for type A aortic dissection in octogenarians. J Thorac Cardiovasc Surg. 2016;152:439-46.

4. Pan E, Gudbjartsson T, Ahlsson A, Fuglsang S, Geirsson A, Hansson EC, et al. Low rate of reoperations after acute type A aortic dissection repair from the Nordic consortium registry. J Thorac Cardiovasc Surg. 2018;156:939-48.
5. Yang B, Malik A, Waidley V, Kleeman KC, Wu X, Norton EL, et al. Shortterm outcomes of a simple and effective approach to aortic root and arch repair in acute type A aortic dissection. J Thorac Cardiovasc Surg. 2018; 155:1360-70.

6. Blum M, Panos A, Lichtenstein SV, Salerno TA. Modified Cabrol shunt for control of hemorrhage in repair of type A dissection of the aorta. Ann Thorac Surg. 1989;48:709-11.

7. Moriyama Y, Iguro Y, Watanabe S, Masuda H, Hisatomi K, Shimokawa S, et al. [Composite valve graft replacement in patients with type A aortic dissection-a modified Cabrol procedure]. Nippon Kyobu Geka Gakkai Zasshi. 1997;45: 1696-700.

8. Cabrol C, Pavie A, Gandjbakhch I, Villemot JP, Guiraudon G, Laughlin L, et al. Complete replacement of the ascending aorta with reimplantation of the coronary arteries: new surgical approach. J Thorac Cardiovasc Surg. 1981;81: 309-15.

9. Chiu P, Miller DC. Evolution of surgical therapy for Stanford acute type A aortic dissection. Ann Cardiothorac Surg. 2016;5:275-95.

10. Chen LW, Wu XJ, Dai XF. Transverse pericardial sinus closure in acute type A aortic dissection operation. Ann Thorac Surg. 2017;104:e351-3.

11. Wu HB, Zhang H, Wang ZW, Hu R, Li LC, Zhang M, et al. Surgery for acute aortic dissection using the Chinese cronus stented elephant trunk technique: experience with 252 patients. J Thorac Cardiovasc Surg. 2014;148: 2132-8.

12. Chen LW, Dai XF, Wu XJ, Liao DS, Hu YN, Zhang H, et al. Ascending aorta and hemiarch replacement combined with modified triple-branched stent graft implantation for repair of acute DeBakey type I aortic dissection. Ann Thorac Surg. 2017; 103:595-601.

13. Mancini MC, Cush EM. Shunt control of bleeding after homograft replacement of the ascending aorta. Ann Thorac Surg. 1999;67:1162-3.

14. Salerno TA, Carvalho EM, Panos AL, Ricci M. Modified Cabrol shunt after complex aortic surgery. Ann Thorac Surg. 2008;86:669-70.

15. Posacioglu H, Apaydin AZ, Calkavur T, Yagdi T, Islamoglu F. Perigraft to right atrial shunt by using autologous pericardium for control of bleeding in acute type A dissections. Ann Thorac Surg. 2002;74:1071-4.

16. Vogt PR, Akinturk H, Bettex DA, Schmidlin D, Lachat ML, Turina MI. Modification of surgical aortoatrial shunts for inaccessible bleeding in aortic surgerymodification of the Cabrol-shunt technique. J Thorac Cardiovasc Surg. 2001; 49:240-2.

Key Words: acute aortic dissection, aortic surgery, modified Cabrol fistula 


\section{APPENDIX E1}

\section{Propensity Score Matching}

A set of covariates was selected to estimate the propensity score (PS) (Table E1). The PS was calculated using logistic regression, and patients in the nonfistula group were matched with patients in the fistula group using the nearest neighbor technique with a predefined caliper of 0.2. Groups were matched in a ratio of 1:1.

After PS matching (Table E1), the chest closure time in the fistula group was shorter than in the nonfistula group.
The volume of postoperative drainage and the amount of red blood cells transfused were less in the fistula group. Also, the fistula group had shorter postoperative mechanical ventilation support period, shorter duration of intensive care unit stay, and lower incidence of postoperative dialysis (Table E2). Thus, similar results were found with PS matching. Because the number of patients and the PS in the 2 groups were similar, and the sample size was small, and PS matching would cause the loss of the sample, PS matching was not used in our study. 
TABLE E1. Preoperative characteristics

\begin{tabular}{|c|c|c|c|c|}
\hline Characteristics & Fistula group $n=76$ & Nonfistula group $n=76$ & $t$ or chi-square test & $P$ value \\
\hline Male & $51(67.1 \%)$ & $51(67.1 \%)$ & 0.00 & 1.00 \\
\hline Age, y & $51.3 \pm 11.0$ & $53.4 \pm 10.8$ & 1.18 & .24 \\
\hline Marfan syndrome & $17(22.4 \%)$ & $15(19.7 \%)$ & 0.16 & .69 \\
\hline Hypertension & $57(75.0 \%)$ & $55(72.4 \%)$ & 0.14 & .71 \\
\hline Diabetes & $5(6.6 \%)$ & $6(7.9 \%)$ & 0.10 & .75 \\
\hline Chronic renal dysfunction & $1(1.3 \%)$ & $2(2.6 \%)$ & & $1.00^{*}$ \\
\hline Previous open heart surgery & $1(1.3 \%)$ & $0(0 \%)$ & & $.44^{*}$ \\
\hline History of cerebrovascular event & $2(2.6 \%)$ & $3(3.9 \%)$ & & $1.00 *$ \\
\hline Acute heart failure & $2(2.6 \%)$ & $1(1.3 \%)$ & & $.59^{*}$ \\
\hline Acute renal dysfunction & $2(2.6 \%)$ & $0(0 \%)$ & & $.19^{*}$ \\
\hline Coronary heart disease & $0(0 \%)$ & $2(2.6 \%)$ & & $.50 *$ \\
\hline Hypoxemia & $4(5.3 \%)$ & $3(3.9 \%)$ & & $.70^{*}$ \\
\hline Prothrombin time, $\mathrm{s}$ & $14.1 \pm 2.1$ & $14.0 \pm 3.1$ & 0.02 & .98 \\
\hline International normalized ratio & $1.1 \pm 0.2$ & $1.2 \pm 0.7$ & 1.33 & .19 \\
\hline Ejection fraction, $\%$ & $63.9 \pm 8.7$ & $62.6 \pm 7.6$ & -0.95 & .34 \\
\hline Pericardial effusion moderate to severe & $8(10.5 \%)$ & $7(9.2 \%)$ & 0.07 & .79 \\
\hline Aortic regurgitation moderate to severe & $25(32.9 \%)$ & $23(30.3 \%)$ & 0.12 & .73 \\
\hline Time from beginning of study to surgery, $\mathrm{m}$ & $14.6 \pm 5.5$ & $16.4 \pm 6.3$ & 1.83 & .07 \\
\hline
\end{tabular}

*Fisher exact test.

TABLE E2. Operative and postoperative data

\begin{tabular}{|c|c|c|c|c|}
\hline Characteristics & Fistula group $n=76$ & Nonfistula group $n=76$ & $t$ or chi-square test & $P$ value \\
\hline \multicolumn{5}{|l|}{ Operative data } \\
\hline Chest closure time, min & $59.3 \pm 13.8$ & $84.2 \pm 28.4$ & 6.88 & $<.0001$ \\
\hline \multicolumn{5}{|l|}{ Postoperative data } \\
\hline Postoperative drainage, $\mathrm{mL}$ & $357.7 \pm 161.1$ & $713.6 \pm 315.3$ & 8.76 & $<.0001$ \\
\hline Reopening for bleeding or oozing & $0(0.0 \%)$ & $7(9.2 \%)$ & & .01 \\
\hline $\mathrm{RBC}$ transfusion, $\mathrm{mL}$ & $497.4 \pm 127.5$ & $764.5 \pm 191.6$ & 10.12 & $<.0001$ \\
\hline Ventilation support, $\mathrm{h}$ & $50.1 \pm 29.2$ & $68.6 \pm 71.3$ & 2.07 & .04 \\
\hline Intensive care unit stay, $d$ & $4.3 \pm 2.7$ & $7.4 \pm 6.2$ & 3.97 & $<.0001$ \\
\hline Dialysis & $1(1.3 \%)$ & $13(17.1 \%)$ & 11.33 & $<.0001$ \\
\hline
\end{tabular}

$R B C$, Red blood cell. 